\title{
Performance Degradation Research of Combined Rotor Considering Rod Relaxation
}

\author{
WANG Ailun ${ }^{1,2, a}$, LUO Zhen ${ }^{1,2, b}$ \\ ${ }^{1}$ State Key Laboratory of High Performance Complex Manufacturing,Central South \\ University,Changsha 410083, China; \\ ${ }^{2}$.School of Mechanical and Electrical Engineering, Central South University, Changsha 410083, \\ China.

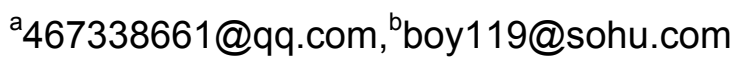

Keywords: rod relaxation; combined rotor; degradation degree; contact interface; natural frequency.

\begin{abstract}
Considering that we have less knowledge of performance degradation mechanism of combined rotor, performance degradation characteristics of combined rotor were discussed, from the perspective of system performance degradation caused by structural damage. Firstly, dynamic features of combined rotor was analyzed which considering rod relaxation and interface contact effect, the relations that natural frequency change with time was obtained. Secondly, defining degradation degree parameter by relative decrease degree of frequency, degradation path was described through dealing with degradation data; degradation assessment for combined rotor was completed as well. Then, unbalance response analysis of combined rotor was conducted according to different degradation degree; degradation characteristics of combined rotor were obtained. Finally, an experimental study on the dynamic characteristics of combined rotor was taken, bending vibration frequency was tested through adjusting the preload of rod, and degradation path was obtained, it confirmed the reasonability of theoretical analysis. The results show that: degradation parameters of combined rotor have exponential distributions, and it has great effect on unbalance response amplitude.
\end{abstract}

\section{Introduction}

In the process of equipment operation, the influence of many aspects, such as as wear, creep, cracking can lead to equipment performance degenerates, which not only reduces the reliability of equipment, but also greatly increases the possibility of equipment failure [1], Performance degradation, in a sense, can be thought of one of the importance reasons that lead to equipment failure. Gas turbine is long served in harsh environments such as high speed, high temperature. To study its performance degradation characteristics and grasp the trend and degree of performance degradation is of great importance to guaranteeing long-term safe operation of gas turbine. Therefore, domestic and foreign scholars have developed many researches on gas turbine performance degradation and have achieved some results. Diakunchak [2] points out that if gas turbine running in extreme environment for a long time, its performance will be gradually degraded over time, and the gas turbine performance degradation is not only related to the running time, but also be affected by the work mode and environment. Rainer Kurz [3] describes in detail the cause of the gas turbine performance degradation and studies the causes of different degradation effects on performance parameters of the gas turbine system. Brook [4] points out that most of the gas turbine energy loss is caused by the compressor performance degradation. Aker [5] studies the effect of gas turbine compressor side partial performance degradation on output power. According to the characteristics of the gas turbine performance degradation, Brothertom [6] proposes that tub curve approximation can be used to describe the gas turbine performance degradation rule, Li, Y.G [7], by using the method of gas path analysis and fitting degradation data, the gas turbine performance degradation curve is obtained, which can be used to predict the residual life of the combustion engine.

All told, aiming at the research of the combined rotor performance degradation caused by creep relaxation, high temperature creep relaxation is the main failure mechanism of fastener. Due to 
combined rotor running under high temperature for a long time and pull rod in the conscription subjected to the threat of creep damage mechanism, the damage will be accumulated to a certain degree, then the pre-tightening force rotor structural integrity will be both reduced, which makes it difficult to maintain normal operation, therefore, the rod stress relaxation is a question that must be considered for the safe operation of the gas turbine. This article studied the performance degradation of combined rod relaxation caused by rod relaxation, which firstly the calculation model considering the combination of the rough interface rotor is established, and then define the amount of degradation index and establish the combined rotor degradation function relation between the quantity and time, thus revealed combined rotor performance degradation mechanism caused by rod relaxation.

\section{Combined rotor calculation model}

Shown in figure 1 is simplified model for a rotor gas turbine's combined compressor rotor (later simplified as combined rotor). The combined rotor is composed of shaft head on both ends, four wheels on the middle and 8 tension rods, which tie rod circumferential Drod's diameter is $90 \mathrm{~mm}$ and six levels of roulette preloaded as a whole. The combined rotor has the following three types of contact: wheel/wheel; rod/wheel through hole wall; rod nut/roulette. According to the engineering practice, the selected combination of rotor material's density is $7800 \mathrm{~kg} \cdot \mathrm{m}^{-3}$ and the elastic modulus is 210GPa. Combined rotor's physical parameters are shown in table 1.

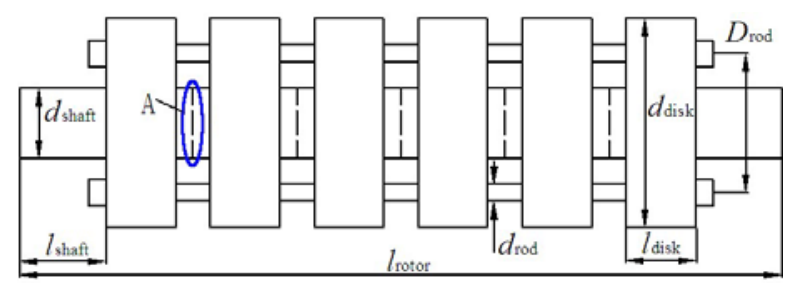

Fig.1 Structure of compressor combined rotor

Table 1 Physical parameters of combined rotor

\begin{tabular}{cc}
\hline Parameter & Value \\
\hline Rotor Length $l_{\text {rotor }} / \mathrm{mm}$ & 380 \\
Disk Lengt $l_{\text {disk }} / \mathrm{mm}$ & 30 \\
Revolving Shaft Length & 50 \\
$l_{\text {shaft }} / \mathrm{mm}$ & 120 \\
Disk Diameter $d_{\text {disk }} / \mathrm{mm}$ & 40 \\
Revolving Shaft diameter & 10 \\
$d_{\text {shaft }} / \mathrm{mm}$ & \\
Rod Diameter $d_{\text {rod }} / \mathrm{mm}$ &
\end{tabular}

\section{The relaxation stress calculation of rod bolts}

Stress relaxation is the kind of phenomenon that the stress reducing accordingly with lower elastic deformation of the connector when the connector's total deformation is changeless. Connectors, although subjected to many factors such as creep and low cycle fatigue, the stress relaxation is the basic mechanism of damage under the steady load condition.

In relaxation, setting $\varepsilon_{0}$ as the initial elastic strain of the connector, under the absolute temperature $\mathrm{T}$, the elastic strain is $\varepsilon_{e}$ and the creep strain is $\varepsilon_{c}$ after time t, c, there is

$$
\varepsilon_{0}=\varepsilon_{e}+\varepsilon_{c}=\sigma / E+\varepsilon_{c}
$$

During which $\mathrm{E}$ is elastic model, to derivative formula (1), there is

$$
\dot{\varepsilon_{\mathrm{c}}}=-\frac{1}{E} \frac{\mathrm{d} \sigma}{\mathrm{dt}}
$$


In view of the relaxation of the pull rod under long-term work, Norton model was used to express the relationship betwwen the creep strain rate and stress:

$$
\dot{\varepsilon_{\mathrm{c}}}=C_{1} \sigma^{C_{2}} e^{\left(-C_{3} / T\right)}
$$

residual stress of rod relaxation is available by combining the points of (2) and (3):

$$
\sigma=\left[\sigma_{0}^{1-C_{2}}-C_{1}\left(1-C_{2}\right) E e^{\left(-C_{3} / T\right)} t\right]^{\frac{1}{1-C_{2}}}
$$

$\sigma_{0}$ is the initial stress, $C_{1}, C_{2}$ and $C_{3}$ are material constants.

Based on the uniaxial creep theory, bar stress $\sigma$ conform to the type:

$$
\sigma=\frac{F_{t}}{A_{\text {rod }}}=\frac{\Delta l}{l_{\text {rod }}} E
$$

In the formula, the $F_{t}$ is the rod preload when the time is $t$, Arod is rod cross-sectional area, $\Delta l$ is the amount of rod preload, $l_{\text {rod }}$ is the total length of tie rod. With reference to the application of gas turbine rod preload in practical engineering and the initial amount prestressing of the rod is defined as one thousandth of the total length of the rod, then $\sigma_{0}=E / 1000$.

GH4169 was applied as the materials of rod of the combined rotor of gas turbine in this article. The material creep experimental data was used to determine the Norton creep model's parameter value, $C_{1}=2.2 \mathrm{E}-009, C_{2}=10.2, C_{3}=50825$. According to reference, using the method of quadratic interpolation, the material elastic modulus of rod, that is $E$ can be received under different temperature $T$. According to the formula (4), to calculate the rod stress relaxation curves under different temperature can be calculated, as shown in figure 2 .

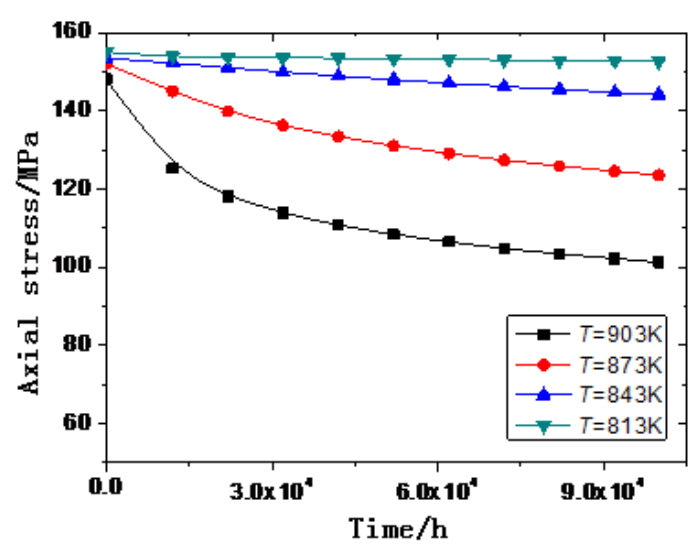

Fig.2 Stress relaxation curve of rod

It can be seen that under the circumstance that the stress less than the yield limit of material, tie rod relaxation is closely related to the working temperature; when the temperature reaches a certain critical value, the rod relaxation will only be obvious. Using the rod, whose material is GH4169 as an example, there will be no rod relaxation under $813 \mathrm{k}$. The rod relaxation is obvious in the initial stage and the relaxation rate is reduced with the extension of time.

\section{Interface stiffness equivalent of combined rotor}

The interface contact stiffness is mainly decided by the pre-tightening force and interface properties. Within the elastic range, the bigger the pre-tightening force is, the larger the stiffness will be; Contact interface roughness, topography and wave degrees are all geometric factors influencing the interface contact stiffness, during which the roughness is the basis for the study of various geometric factors. The contact interface of the model adopted in the research only considers the effect of roughness and view this as the basis to calculate the relationship between contact stiffness and pre-stressing force and to conduct the equivalent contact stiffness. 


\section{Determination of interface stiffness}

This article uses the calculation method of combining the infinitesimal body model analysis which have the same interface roughness and the macro dimension and thus accurately get the contact stiffness between wheel, the steps are as follows: (1) calculate the area S1 of contact interface between heels ( as shown in figure 1 ), stress analysis was carried out on the wheel contact interface, the interface pressure $\mathrm{p}$ was then got after preloaded; (2) establish a infinitesimal body model when the contact interface are is $\mathrm{s} 2$, the roughness is $0.4 \mathrm{~m}$ and the pressure is $\mathrm{p}$ according to the method put forward in literature (figure 3), and conduct the stress and strain analysis to this model and extract the contact stiffness kn; (3) Takashi Yoshimura points out that as long as the average contact pressure is the same, the dynamic performance data in combination with surface per unit area is the same, therefore, on the basis of getting the contact stiffness of the micro finite element model, the interface contact stiffness $\mathrm{k}$ of the combined rotor under the effect of pre-stressing force $\mathrm{F}$ could be got using formula (6) and (7) (figure 4).
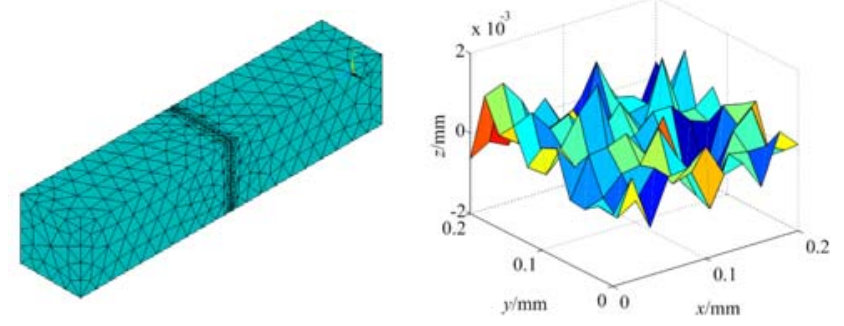

Fig.3 Micro-scale finite element model

$$
\begin{aligned}
& F=P \times S_{1} \\
& K=\frac{S_{1}}{S_{2}} k_{\mathrm{n}}
\end{aligned}
$$

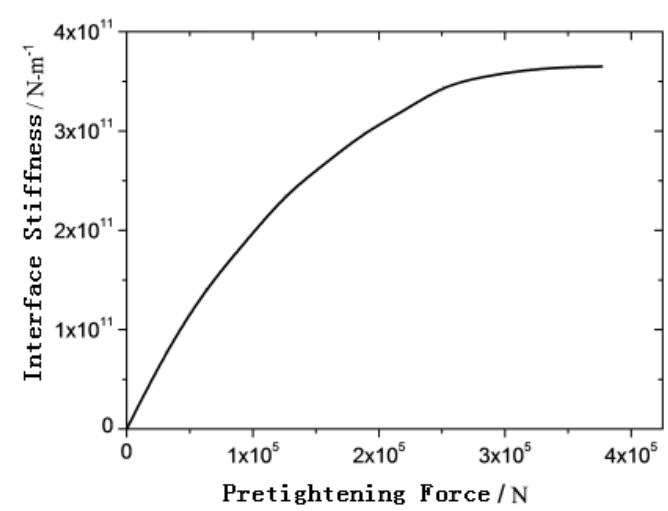

Fig.4 Stiffness of interface versus pretension for combined rotor

\section{Interface stiffness equivalent}

General finite element software can be applied to conduct statics analysis on the contact, while contact belongs to nonlinear problem and direct dynamics analysis could not be carried out contact interface considering roughness. Therefore, when conduct the dynamic analysis, the contact interface equivalent should be carried on. The joint surface that under external loading, when the stiffness is known, according to the strain energy equivalent principle, the contact interface stiffness is equivalent for the elastic modulus $\bar{E}$ of virtual material layer, the equivalent formula is:

$$
\bar{E}=K h / S_{1}
$$

$\mathrm{h}$ is the thickness of the virtual material layer. 


\section{The analysis of combined rotor's inherent characteristic}

In view of the combined rotor shown in figure 1, conducting dynamics characteristic analysis of combined rotor considering contact interface and establishing the relation between the combined rotor's inherent characteristic with the time, the steps are as follows: 1. get the rod stress relaxation law, by formula (4) and get pre-stressing force Ft of the rotor under different time combining formula (5); 2. Use the relation between the interface stiffness and the pre-pressing force that shown in figure 3 to equivalent the wheel interface stiffness by different pre-pressing force through elastic modulus of virtual material layer.

For the study of composite rotor dynamic characteristics, first of all, with the structure characteristics of a composite rotor, combined rotor dynamics model is established. According to the above analysis, equivalent to consider the roughness of the contact interface, the rough equivalent for the virtual material layer interface, contact stiffness equivalent for the virtual material layer modulus of elasticity, the pull rod unit is added to the system as attachment, can consider the combination of the contact interface roughness of rotor dynamics model is set up as shown in figure 5.

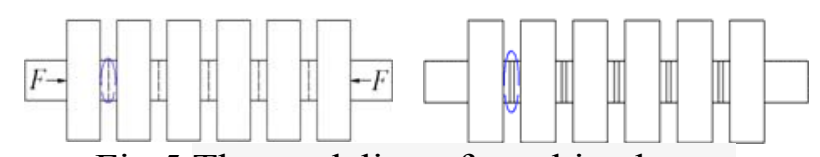

Fig. 5 The modeling of combined rotor

This paper only analyzes the bending vibration feature set of the rotor,Shear stiffness of the interface can not be considered,take the virtual layer thickness of $0.5 \mathrm{~mm}, v=0$.Operating temperature of the combined rotor is $873 \mathrm{~K}$. When performing the combined rotor dynamics analysis, the contact interface between the rod and the wheel bonding,material layer at both ends of the virtual link with the wheel using Ansys multi-point constraint technology, the virtual layer of material with fine mesh.Table 2 lists the combinations of natural frequencies of the rotor at different times.For further analysis of the inherent characteristics of the combined rotor, reducing the amount of the relative frequency is defined as follows:

$$
\eta=\frac{f_{0}-f_{t}}{f_{0}}
$$

Where, $\mathrm{f} 0$ is the decrease amount of combined rotor frequency at initial time, $\mathrm{ft}$ is the decrease amount of combined rotor frequency at time $t$ 。

Table 2 shows that with the increase of the relaxation time, the combined rotor natural frequency drift.The relationship of decrease amount of combined rotor frequency and time shown in Figure 6.

Tab.2 Natural frequency of combined rotor at different time

\begin{tabular}{cccc}
\hline $\begin{array}{c}\text { Time } \\
t / \mathrm{h}\end{array}$ & $\begin{array}{c}\text { First order } \\
f_{t / \mathrm{Hz}}\end{array}$ & $\begin{array}{c}\text { Second order } \\
f_{t} / \mathrm{Hz}\end{array}$ & $\begin{array}{c}\text { Third order } \\
f_{t} / \mathrm{Hz}\end{array}$ \\
\hline 0 & 530.02 & 1135.2 & 2202.9 \\
10000 & 528.47 & 1131.3 & 2195.8 \\
20000 & 527.79 & 1129.9 & 2193.2 \\
30000 & 527.31 & 1129.1 & 2191.5 \\
40000 & 527.03 & 1128.6 & 2190.6 \\
50000 & 526.83 & 1128.1 & 2190.0 \\
60000 & 526.67 & 1127.8 & 2189.4 \\
70000 & 526.51 & 1127.4 & 2188.9 \\
80000 & 526.36 & 1127.1 & 2188.4 \\
90000 & 526.31 & 1126.9 & 2188.0 \\
10000 & 526.28 & 1126.8 & 2187.7 \\
\hline
\end{tabular}




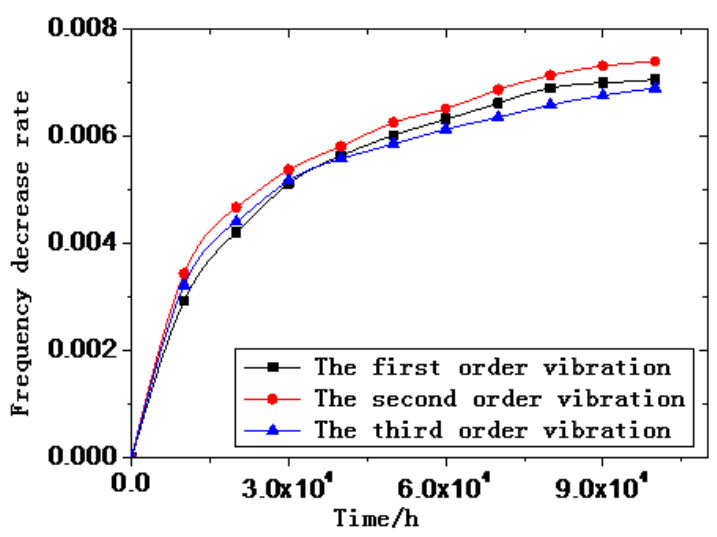

Fig.6 Relative decrease degree of combined rotor frequency

\section{Degradation track describe of combined rotor}

In order to determine the degradation of the equipment, usually selected key performance parameters as performance degradation parameters, The existing Study on the degradation of gas turbine selects machine performance parameters (power, pressure ratio) as degradation parameters. These parameters of machine performance in combination with the rotor machine performance degradation is not directly related.It is not suitable as the amount of degradation indicators of combination rotor. In this paper,it selects the combination of the rotor relative frequency reducing amount $(\eta)$ as a amount of combined rotor performance degradation caused by the relaxation parameters of the rod, the value of each parameter about amount of degradation is degraded data, the degradation data obtained by curve fitting can give parameters changing over time function, the degradation paths(Figure 7)to give an exponential function distribution of combination of the rotor (the operating temperature of $873 \mathrm{~K}$ ) obeying the formula of degradation paths (10) .

$$
D=D_{1} e^{\left(-t / D_{2}\right)}+D_{3}
$$

Where, $\mathrm{D}$ is the amount of degradation,t is trolley relaxation time, degradation parameters $D_{1} 、 D_{2}$ 、 $D_{3}$ are $-0.0068 、 23039 、 0.0071$ respectively.

Analysis of the composite rotor degradation data at different temperatures, been assembled the degradation paths of composite rotors under different temperature as figure 8. As can be seen,within a certain time frame,as the relaxation time increases, the degradation of composite rotor increases; Degradation of the composite rotor is sensitive to temperature, and in the same time, the higher the temperature, the greater the degradation of the composite rotor.Therefor,in order to improve the reliability of the composite rotor, reduce the amount of degradation of the composite rotor, the temperature lever should try to be reduced during the operation time.

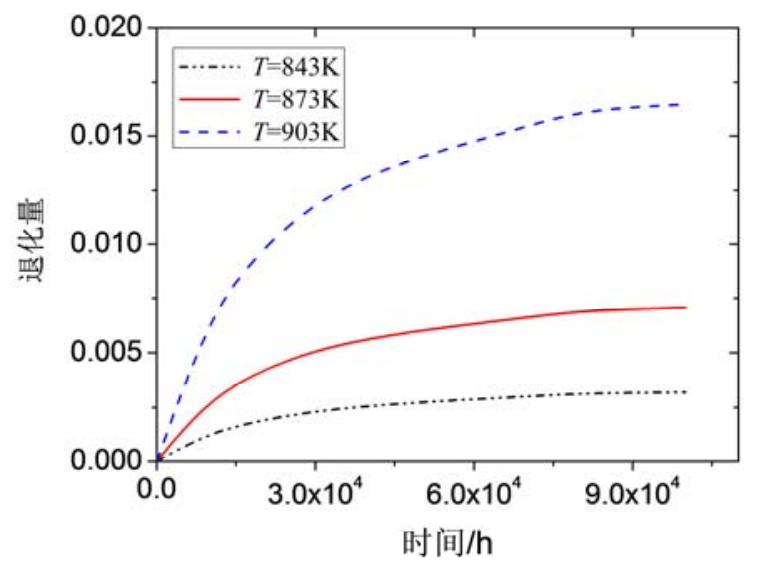

Fig.8 Degradation path of combined rotor at different temperature 


\section{Summary}

1)In case of stress is less than the yield limit, relaxation of the lever is closely related to operating temperature. When the temperature reaches a certain critical value, the relaxation of the rods is more obviously. Rod material for GH4169 as an example,the relaxation does not occur below the critical temperature at $813 \mathrm{~K}$; Rod relaxation at the initial stage is more noticeable, with the extension of time, the relaxation rate decreases.

2)As the relaxation time increases, the intrinsic frequency of the composite rotor drifted.At the same time, the combination of lower rotor intrinsic frequency distributions with different, first-order, second-order bending vibration of bending vibration, third-order bending frequency increased.The natural frequencies of composite rotor at different times, distribution value of similar value, relatively the lower volume of frequency showed an increasing trend over time.Table 1 Three Scheme comparing

\section{Foundation items:}

National key basic research and development program (973 Program)

Code:2013CB035706

\section{References}

[1] Lee J. Measurement of machine performance degradation using a neural network model[J]. Computers in Industry, 1996, 30(3):193-209.

[2] Diakunchak, I.S., Performance degradation in industrial gas turbines[J], ASME J. of Engineering for Gas Turbines and Power, 1992, 114:161-168.

[3] Brooks, F. J. GE Gas Turbine Performance Characteristics[R], 2003, GE Power Systems GER-3567H.

[4] Aker, G. and Saravanamuttoo, H., Predicting gas turbine performance degradation due to compressor fouling using computer simulation techniques[J], Journal of Engineering for Gas Turbines and Power, 1989,111(2):343-350.

[5] Rainer Kurz, Klaus Brun. Degradation in gas turbine systems[J]. Journal of Engineering for Gas Turbines and Power, 2001,123(1):70-77.

[6] Brotherton T., Jahns G., Jacobs J,et al. Prognosis of Fault in Gas Turbine Engines[C], IEEE Aerospace Conference Proceedings, 2000(6):163-171.

[7] Li Y G, Nilkitsaranont P. Gas turbine performance prognostic for condition-based maintenance[J]. Applied Energy, 2009(86):573-584 